\title{
The ethnobotany of Ngusaba ceremonial plant utilization by Tenganan Pegringsingan community in Karangasem, Bali, Indonesia
}

\author{
DEWA AYU SRI RATNANI ${ }^{1, \bullet}$, I KETUT JUNITHA ${ }^{2}$, ENIEK KRISWIYANTI ${ }^{2}$, I NYOMAN DHANA ${ }^{3}$ \\ ${ }^{1}$ Program of Biology Education, Faculty of Teacher Training and Education, Universitas Mahasaraswati Denpasar. J1. Kamboja No. 11A, Denpasar \\ 80233, Bali, Indonesia. Tel.: +62-361-227019, ^email: sri.ratnani67@gmail.com \\ ${ }^{2}$ Program of Biology, Faculty of Mathematics and Natural Sciences, Universitas Udayana. Jl. Raya Kampus Unud No. 9, Jimbaran, Badung 80361, Bali, \\ Indonesia \\ ${ }^{3}$ Program of Anthropology, Faculty of Humanities, Universitas Udayana. Jl. Nias No. 13, Sanglah, Denpasar 80114, Bali, Indonesia
}

Manuscript received: 22 March 2021. Revision accepted: 28 March 2021.

\begin{abstract}
Ratnani DA, Junitha IK, Kriswiyanti E, Dhana IN. 2021. The ethnobotany of Ngusaba ceremonial plant utilization by Tenganan Pegringsingan community in Karangasem, Bali, Indonesia. Biodiversitas 22: 2078-2087. Tenganan Pegringsingan is an ancient village in Bali, Indonesia, which often performs several ceremonies with high intensity. One of them is the Ngusaba ceremony, where many plants are utilized both in species and quantity. Hence, this study aimed to identify the species, family, local names, sources, and parts of plants, used for Ngusaba ceremonies by the Tenganan Pegringsingan community including the Index of Cultural Significance (ICS). Data analysis was qualitative and quantitative. Furthermore, the qualitative method was used to obtain data on the plants' local names, while snowball sampling was applied to select key informants through in-depth interviews and moderate participation. The results showed that the 130 species distributed in 56 families mostly belonging to the purchased source (34.61\%). The Poaceae is the largest family, while the most widely used part of the plant is the leaf. Based on the ICS analysis results, a range of 2-114 values was obtained. The highest value is Base (Piper betle L.) and kangkung (Ipomoea batatas L.) as lowest.
\end{abstract}

Keywords: Ancient villagers, local knowledge, Ngusaba plant

\section{INTRODUCTION}

Bali is one of the tourism destinations in Indonesia has many attractions. Its distinctive feature is a unique blend of humans, nature, and culture, including customs and religious ceremonies where plants play an important role. Plants or their parts are the most important elements in material associated with the Yadnya ceremonies (Sujarwo 2020), including the ngusaba ceremony. The Ngusaba ceremony is a social activity to connect with the all mighty God (Ida Sang Hyang Widhi), which also includes banquets and subak village thanksgiving (Arwati 2007). It provides much information about the use of many plants or their parts, including leaves, flowers, fruits, seeds, and tubers (Adiputra 2011).

The utilization of Ngusaba by the Tenganan Pegringsingan community has some problems: which include (i) many of the ceremonial ingredients types and quantities needed exceed these plant's availability in nature; (ii) Only a few people are interested in traditional practices such as agriculture, because most of them rely on tenant farmers; (iii) The existence of plants, especially endemic species become increasingly hard to be found. Besides, plants are an important source of food, medicine, spice, construction materials, etc. in rural areas (Sujarwo et al. 2016; Sujarwo dan Caneva 2016; Sujarwo dan Keim 2017; Navia et al. 2020). They have many cultural sides, namely history, religion, language, art, politics, and social structure (Kakudidi 2004). They also have an important meaning, especially in various religious ceremonies
(Helida et al. 2015; Ristanto et al. 2020). Several plants are part of various ritual purposes (Sharma and Pegu 2011; Iskandar and Iskandar 2017) and a source of livelihood for the local people (Suwardi et al. 2020) that believe ritual is one of the most important instruments for understanding local communities and offering, to conserve nature (Geng et al. 2017). The conservation of plant resources is very important to combine with the understanding and awareness of local communities' cultural practices (Sheybani et al. 2015; O'Neill et al. 2017).

However, information technology development and modern lifestyle have led to a decline in local communities' traditional knowledge (Putri et al. 2017) and this condition also affects the Tenganan Pegringsingan community. In addition, the knowledge of ritual plant utilization is diminishing because it is only passed across generations orally and has remained unwritten (Anderson et al. 2011; Surata et al. 2015; Nisyapuri et al. 2018). The loss of local knowledge implicates plant resources' existence, as well as triggers disease and professional changes (Gomez et al. 2010; Cuadra et al. 2012; Ju et al. 2013; Vásquez et al. 2016; Aswani et al. 2018). The knowledge is very useful to conserve biodiversity, hence it needs to be maintained (Yusro et al. 2014) and documented for good management to halt the menace of biodiversity depletion (Adom 2018). There has been much effort in biodiversity conservation, such as plant preservation and documentation of their utilization through ethnobotany which is the study of utilitarian relationships between humans and plants in natural ecosystems and other social components (Hakim 
2014). Ethnobotany data cover botany, taxonomy, and regional botanical knowledge. It is also essential for biodiversity conservation (Pieroni et al. 2014; Tapundu and Anam 2015), fulfillment of needs such as food, health, and culture (Setiawan and Qiptiyah 2014; Tamalene et al. 2016; Mesfin et al. 2018), construction, decoration, and other living necessities (Bosworth et al. 2011). Today, ethnobotany has become a crucial study area, which covers management resource development, biodiversity conservation at the genetic, species and ecosystem level, and regional socioeconomic development (Caneva et al. 2017). Therefore, this study aims to identify plants used for Ngusaba ceremonies by the Tenganan Pegringsingan community.

\section{MATERIALS AND METHODS}

\section{Study area}

This study was conducted in Tenganan Pegringsingan community of Tenganan Village, Manggis Subdistrict, Karangasem District, Bali, Indonesia, from February to August 2020. The location is at positions $8000^{\circ} .00^{\prime}$ to $8041^{\circ} .37 .8^{\prime} \mathrm{S}$ and $115035^{\circ} .9 .8^{\prime}$ to $115054^{\circ} 8.9^{\prime} \mathrm{E}$, at an altitude of 70-400 $\mathrm{m}$ asl. The village's temperatures ranging from $28-31^{\circ} \mathrm{C}$.

\section{General description of the study sites}

Tenganan Pegringsingan is located in Manggis Subdistrict, Karangasem District, with a distance of \pm 20 $\mathrm{km}$ from the District City, and $\pm 68 \mathrm{~km}$ from Denpasar. It is physiographically surrounded by three-quarters of a circle of hills forming borders in the north as Macang Village (kaja hill), east as Asak Village (kangin hill), and west as Ngis Village (kauh hill), but directly adjacent to Pesedahan Village in the south. According to usage the area includes paddy rice lands covering 255.85 ha, drylands covering 480.89 ha, and Adat forest lands covering 197.32 ha. (Monograph of Tenganan Village 2020). Tenganan Pegringsingan total population is 1022 , with the family heads being 338, while the location map is shown in Figure 1.

\section{Informant selection}

Key informants were consulted with community leaders and selected using the snowball sampling technique, which was carried out in a chain by questioning those that have been interviewed or contacted previously (Hariyadi and Ticktin 2012). Furthermore, they had much information about the Ngusaba ceremony (Nurdiani 2014), including the offering expert, ceremony officials, and community leaders.
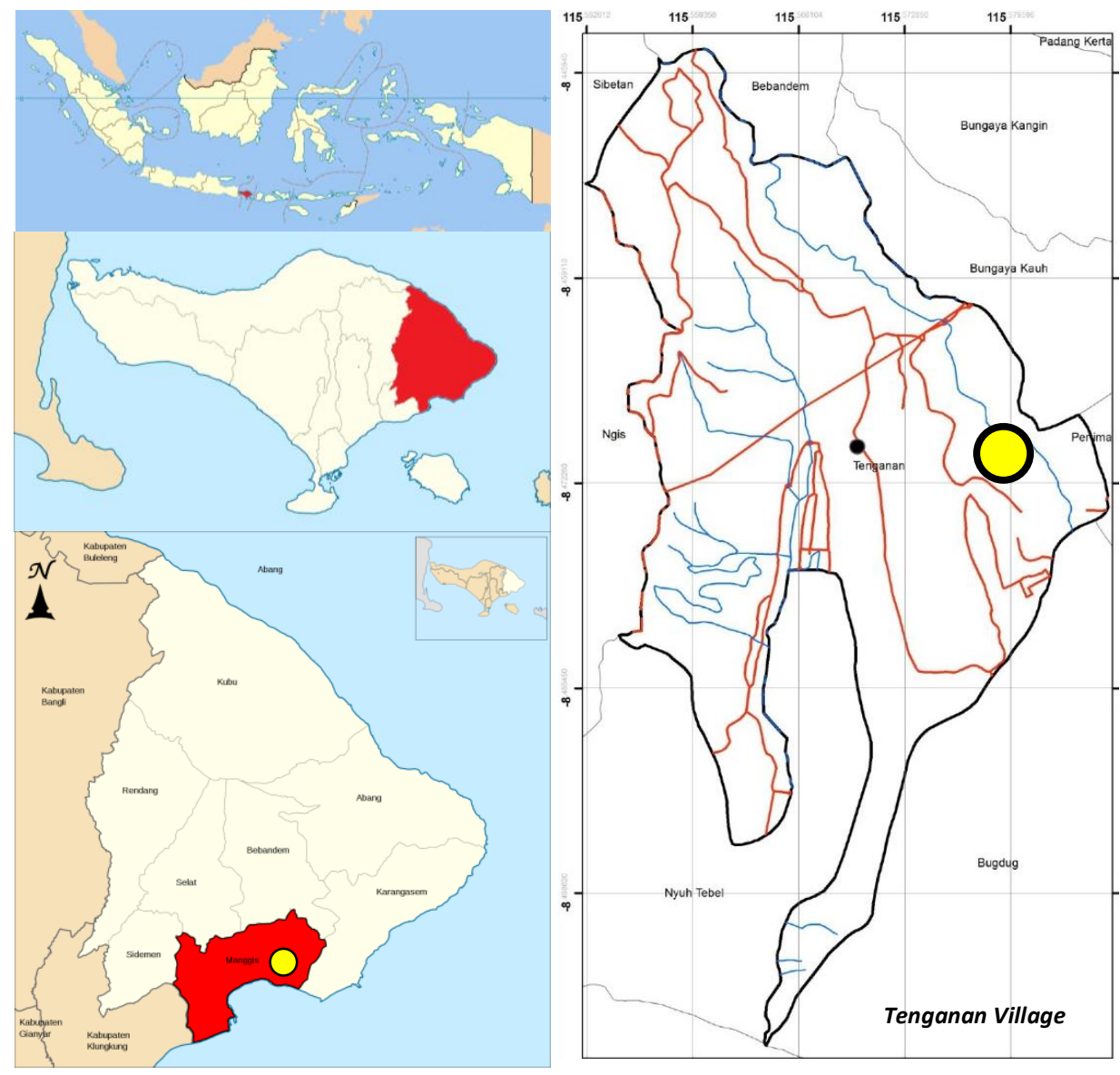

Figure 1. Map of the location of Tenganan Pegringsingan community $(O)$ in Tenganan Village, Manggis Subdistrict, Karangasem District, Bali, Indonesia (Monograph of Tenganan Village 2020) 


\section{Data collection}

Ethnobotany data were collected through semistructured interviews and moderate participation in the form of species, family, local names, parts, sources, and the Index of Cultural Significance (ICS) of plants, which were analyzed qualitatively and quantitatively. A descriptive narrative was carried out for qualitative analysis through data reduction, display and analysis (Sugiyanto 2017). The quantitative analysis of the Ngusaba ceremonial plant was carried out through the ICS from Purwanto (2003). The ICS showed the importance values of each useful plant species based on the community's needs, and its calculation results showed each plant's importance level. The equation provided is to be employed to calculate ICS.

$$
\operatorname{ICS}=\sum_{i=1}^{n}(q x \text { i } x \text { e e }) \text { ni }
$$

Because each species of plant has several uses, the equation is as follows:

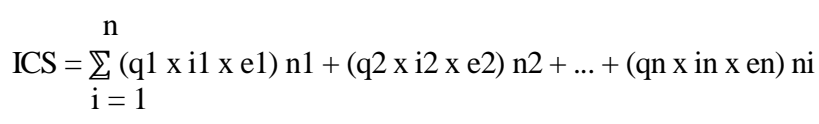

Where:

ICS $=$ the number of calculations the utilization of a plant species from 1 to $\mathrm{n}$,

$\mathrm{q}$ : quality value calculated by giving a score or value on the quality value of a plant species: $3=$ the main Ngusaba ceremony ingredient; 2 = additional Ngusaba ceremony materials + primary materials, $1=$ other Ngusaba ceremony materials + secondary materials + primary materials

$\mathrm{i}$ : intensity value describes the intensity of utilization of useful plant species by giving values: value $3=$ high intensity; $2=$ moderate intensity; $1=$ low intensity.

e : exclusivity value: value $2=$ most important, is the first choice and is second to none; $1=$ possibility of being a choice of secondary materials (Turner 1998; Purwanto 2003; modification of researchers).

The plants were collected with the informants and then identified by matching with the herbarium specimen of the Bali Botanical Garden, the picture on the flora book, and images on plantNet. Their scientific names were verified using online sources (e.g. The Plantlist 2019).

\section{RESULTS AND DISCUSSION}

\section{Types of plants utilized for Ngusaba ceremony}

The results showed 11 types of Ngusaba ceremonies carried out by the Tenganan Pegringsingan community, including Ngusaba Kasa, Karo, Ketiga, Kelima (sambah), Kenem, Kepitu, Kaulu, Kesanga, Kedasa, Desta, and Sada. The ceremonies are held almost monthly every year, and each lasts for three days, except for sambah which lasts for one month. The Ngusaba plants in Tenganan Pegringsingan Village have a high diversity of 130 species belonging to 56 families among which the largest is Poaceae (16 species), followed by Fabaceae (9) and Musaceae (8). The percentage of the ngusaba plant families utilized by the community is shown in figure 2 . The various species were collected from various habitats, mainly wild vegetation in the forest, roadsides, in front of the house, home gardens and drylands. The growth form indicated that the most widely used ngusaba are obtained from herbs (57 species or $43.84 \%$ ), followed by trees (38 species or $29.23 \%$ ), and shrubs (34 species or $26.15 \%$ ) (Table 1).

This result is higher than 26 species representing 17 families found to be commonly used for performing the six main traditional rituals of the Karangwangi people (Erawan et al. 2018). The Baduy community uses 50 species representing 28 families for nine stages of their pure agricultural activity (Iskandar and Iskandar 2017), while the Aceh tribe in Peureulak uses 51 species consisting of 47 genera and 34 families (Sutrisno et al. 2020). Moreover, Bali Aga village uses 125 plant species for all the Panca yadnya ceremony (Sujarwo 2020) and based on these, cultural diversity shows biodiversity. The diversity of plants used for Ngusaba ceremonial offerings is an expression of the region's uniqueness which is a mountainous area surrounded by hills. A region's uniqueness determines biodiversity, including plants in a specific ecosystem. Each ethnic group grows according to regional uniqueness, culture, and natural resources' availability (Suryadarma 2017). Almost all the Poaceae family plants used for Ngusaba ceremonies are edible, staple foodstuffs, and the main agricultural product.

\section{Plant parts utilized for Ngusaba ceremony by Tenganan Pegringsingan community}

The plant parts used are in the form of leaf, stem, flower, fruit, seeds, tuber, and rhizome as presented in Figure 3. The most widely used are leaves, while the rhizome is the lowest.

The most utilized parts reported were leaves $(45.52 \%)$, followed by fruits $(38.80 \%)$ and flowers $(17.91 \%)$. Other studies such as Mesfin et al. (2013), Riadi et al. (2019), and Ristanto et al. (2020) also reported that leaves were the most commonly used. The high utilization of Ngusaba leaves appears to be associated with several advantages such as higher number or productivity of leaves that are easier to obtain than the other parts (Handayani 2015). Piper betle L. leaves are mostly utilized in all types of Ngusaba ceremonies. These are made in various forms that differentiate their names and are also irreplaceable (exclusive) and a must have in every offering. Furthermore, banana shoots are used almost equally as Piper betle L. and those having leaves that are useful to local people are included in a taste of sepia banana group, where the most widely used is Musa acuminata L. (biyu keladi). 
Table 1. Species of Ngusaba plants utilization by Tenganan Pegringsingan community

\begin{tabular}{|c|c|c|c|c|c|}
\hline Family/scientific name & Local name & Plants part & Habitus & ICS value & Category \\
\hline \multicolumn{6}{|l|}{ Acanthaceae } \\
\hline Asystasia gangetica $\mathrm{L}$. & Loja & Leaf & Herb & 6 & Low \\
\hline Graptophylum pictum $\mathrm{L}$. & Temen & Leaf & Shrub & 24 & Moderate \\
\hline Justicia adhatoda L. & Dausa & Leaf & Shrub & 102 & Very high \\
\hline Thunbergia erecta Benth & Terom Pelung & Flower & Shrub & 4 & Very low \\
\hline \multicolumn{6}{|l|}{ Achariaceae } \\
\hline Pangium edule Reinw. & Pangi & Seed & Tree & 20 & Moderate \\
\hline \multicolumn{6}{|l|}{ Agavaceae } \\
\hline Dracaena marginata Lam. & Sumenek & Leaf & Tree & 24 & Moderate \\
\hline \multicolumn{6}{|l|}{ Amaranthaceae } \\
\hline Celocia cristata $\mathrm{L}$. & Kenyiwaan & Flower & Herb & 6 & Low \\
\hline Allium sativum $\mathrm{L}$ & Kesuna & Tuber & & \multicolumn{2}{|c|}{ Amaryllidaceae } \\
\hline Allium cepa L. & Bawang & Tuber & $\begin{array}{l}\text { Herb } \\
\text { Herb }\end{array}$ & $\begin{array}{l}42 \\
30\end{array}$ & $\begin{array}{l}\text { Moderate } \\
\text { Moderate }\end{array}$ \\
\hline \multicolumn{6}{|l|}{ Anacardiaceae } \\
\hline Mangifera caesia Jack. & Wani & Fruit & Tree & 12 & Low \\
\hline Mangifera indica $\mathrm{L}$ & Poh Arum Manis & Fruit & Tree & 12 & Low \\
\hline Mangifera indica $\mathrm{L}$ & Poh Madu & Fruit & Tree & 12 & Low \\
\hline Mangifera odorata Griff. & Pakel & Leaf & Tree & 6 & Low \\
\hline \multicolumn{6}{|l|}{ Annonaceae } \\
\hline Cananga odorata Lamk. & Sandat & Flower & Tree & 12 & Low \\
\hline \multicolumn{6}{|l|}{ Apocynaceae } \\
\hline Plumeria alba L. & Jepun Bali & Flower & Tree & 24 & Moderate \\
\hline Plumeria alba $\mathrm{L}$. & Jepun Cenana & Flower & Tree & 4 & Very low \\
\hline Plumeria acuminata L. & Jepun Merah & Flower & Tree & 4 & Very low \\
\hline Allamanda cathartica $\mathrm{L}$. & Kecubung Kuning & Flower & Shrub & 4 & Very low \\
\hline \multicolumn{6}{|l|}{ Araceae } \\
\hline Colocasia esculenta Schott. & Keladi & Leaf, tuber & Herb & 12 & Low \\
\hline \multicolumn{6}{|l|}{ Araliaceae } \\
\hline $\begin{array}{l}\text { Schefflera elliptica (Blume) Harms. } \\
\text { Arecaceae }\end{array}$ & Kayu Belang & Leaf & Shrub & 20 & Moderate \\
\hline Arenga pinnata Merr & Jaka & Midrib, leaf, fruit & Tree & 66 & High \\
\hline Areca catechu & Buah & Fruit, flower & Tree & 66 & High \\
\hline Cocos nucifera $\mathrm{L}$ & Nyuh Gadang & Midrib, leaf, fruit & Tree & 84 & High \\
\hline Cocos nucifera L & Nyuh Barak & Midrib, leaf, fruit & Tree & 84 & High \\
\hline Salacca zalacca $\mathrm{L}$ & Salak & Fruit & Tree & 12 & Low \\
\hline \multicolumn{6}{|l|}{ Asclepiadaceae } \\
\hline $\begin{array}{l}\text { Hoya australis R.Br.ex.Trail. } \\
\text { Asteraceae }\end{array}$ & Tebel-tebel & Leaf & Herb & 6 & Low \\
\hline Tagetes erecta $\mathrm{L}$. & Gumitir & Flower & Herb & 4 & Very low \\
\hline Tithonia aristrata Oerst. & Sungenge & Flower & Herb & 6 & Low \\
\hline \multicolumn{6}{|l|}{ Athyriaceae } \\
\hline \multicolumn{6}{|l|}{ Bromeliaceae } \\
\hline Ananas comusus Mer. & Manas & Fruit & Herb & 12 & Low \\
\hline \multicolumn{6}{|l|}{ Cactaceae } \\
\hline Hylocereus polyrhizus Britton\&Rose & Buah Naga & Fruit & Herb & 26 & Moderate \\
\hline \multicolumn{6}{|l|}{ Clusiaceae } \\
\hline Calophyllum inophyllum $\mathrm{L}$ & Camplung & Leaf & Tree & 6 & Low \\
\hline Mesua ferrea $\mathrm{L}$. & Nagasari & Leaf & Shrub & 6 & Low \\
\hline Garcinia mangostana $\mathrm{L}$. & Manggis & Fruit & Tree & 12 & Low \\
\hline \multicolumn{6}{|l|}{ Combretaceae } \\
\hline Lumnitzera littorea Jack. & Padi-padi & Leaf & Tree & 6 & Low \\
\hline Convolvulaceae & & & & & \\
\hline Ipomoea aquatica Forssk. & Kangkung & Stem, leaf & Herb & 2 & Very low \\
\hline Cucurbitaceae & & & & & \\
\hline Citrulus lanatus (Thunb.) & Semangka & Fruit & Herb & 24 & Moderate \\
\hline Cucumis sativus $\mathrm{L}$. & Ketimun & Fruit & Herb & 30 & Moderate \\
\hline Dioscoreaceae & & & & & \\
\hline Dioscorea bulbifera L. & Ubiaung buluh & Tuber & Herb & 6 & Low \\
\hline
\end{tabular}




\begin{tabular}{|c|c|c|c|c|c|}
\hline \multicolumn{6}{|l|}{ Euphorbiaceae } \\
\hline Aleurites moluccanus $\mathrm{L}$. & Tingkih & Seed & Tree & 18 & Low \\
\hline Codiaeum variegatum & Kayu Mas & Leaf & Shrub & 24 & Moderate \\
\hline Phyllanthus boxifolius Muell.Arg. & Kayu Sisi & Leaf & Shrub & 24 & Moderate \\
\hline \multicolumn{6}{|l|}{ Fabaceae } \\
\hline Caesalpinia pulcherima (L) Sw. & Sumerak & Leaf, flower & Shrub & 24 & Moderate \\
\hline Casia glauca Lamk. & Kembang Kuning & Leaf & Shrub & 78 & High \\
\hline Clitoria ternatea $\mathrm{L}$. & Teleng & Flower & Shrub & 12 & Low \\
\hline Erythrina abyssinica Lam. & Dapdap & Leaf, stem & Tree & 36 & Moderate \\
\hline Psophocarpus tetragonolobus L. & Kacang Botor & Seed & Herb & 6 & Low \\
\hline Tamarindus indica Linn. & Cagi & Seed & Tree & 6 & Low \\
\hline Indigofera tinctoria Mill. & Taum & Leaf & Shrub & 6 & Low \\
\hline Vigna unguiculata L. & Kacang Barak & Seed & Herb & 30 & Moderate \\
\hline Vigna radiata $\mathrm{L}$ & Kacang Ijo & Seed & Shrub & 6 & Low \\
\hline \multicolumn{6}{|l|}{ Heliconiaceae } \\
\hline Heliconia stricta Huber. & Pisang Ikik & Leaf, fruit & Herb & 6 & Low \\
\hline \multicolumn{6}{|l|}{ Hydrangeaceae } \\
\hline Hydrangea macrophylla $\mathrm{L}$. & Bunga Biru & Flower & Shrub & 4 & Very low \\
\hline \multicolumn{6}{|l|}{ Lamiaceae } \\
\hline Ocimum gratissimum $\mathrm{L}$. & Sulasih & Leaf & Shrub & 6 & Low \\
\hline \multicolumn{6}{|l|}{ Leeaceae } \\
\hline Leea aculeata Burm.f. & Girang & Leaf, stem & Shrub & 4 & Very low \\
\hline Leea indica Burm.f & Kelawasan & Leaf & Shrub & 24 & Moderate \\
\hline \multicolumn{6}{|l|}{ Leguminosae } \\
\hline Bauhinia purpurea L. & Badya & Leaf & Tree & 6 & Low \\
\hline Indigofera tinctoria Mill. & Kumaligi & Leaf & Herb & 24 & Moderate \\
\hline Mucuna pruriens Wilmot. & Juleh & Seed & Herb & 6 & Low \\
\hline Lablab purpureus L. & Komak selem & Seed & Herb & 12 & Low \\
\hline \multicolumn{6}{|l|}{ Lygodiaceae } \\
\hline Lygodium circinatum (Burm.f) Sw. & Ata & Stem+leaf & Herb & 36 & Moderate \\
\hline \multicolumn{6}{|l|}{ Liliaceae } \\
\hline Cordyline fruticosa $\mathrm{L}$. & Andong & Leaf & Herb & 30 & Moderate \\
\hline \multicolumn{6}{|l|}{ Limnocharitaceae } \\
\hline Limnocharis flava $\mathrm{L}$. & Biyah & Leaf & Herb & 6 & Low \\
\hline \multicolumn{6}{|l|}{ Malvaceae } \\
\hline Durio zibethinus L. & Duren & Fruit, leaf & Tree & 12 & Low \\
\hline Hibiscus rosasinensis L. & Pucuk Bang & Flower & Shrub & 84 & High \\
\hline \multicolumn{6}{|l|}{ Magnoliaceae } \\
\hline Michelia champaca $\mathrm{L}$. & Cempaka & Flower & Shrub & $12 \mathrm{t}$ & Low \\
\hline \multicolumn{6}{|l|}{ Marantaceae } \\
\hline Maranta ramosissima Wall. & Kecandik & Leaf & Herb & 6 & Low \\
\hline \multicolumn{6}{|l|}{ Meliaceae } \\
\hline Aglaia odorata Lour. & Kiulan & Flower & Shrub & 4 & Very low \\
\hline Azadirachta indica Juss & Apah & Leaf & Tree & 30 & Moderate \\
\hline Lansium domesticum $\mathrm{L}$. & Langsat & Fruit & Tree & 4 & Very low \\
\hline Moraceae & & & & & \\
\hline Ficus benyamina $\mathrm{L}$. & Bingin & Leaf & Tree & 24 & Moderate \\
\hline Ficus religiosa $\mathrm{L}$. & Ancak & Leaf & Tree & 8 & Low \\
\hline Musaceae & & & & & \\
\hline Musa paradisiaca $\mathrm{L}$. & Biyu Gedang Saba & Leaf, fruit & Herb & 30 & Moderate \\
\hline Musa acuminata L. & Biyu Ketip Tulang & Leaf, fruit & Herb & 30 & Moderate \\
\hline Musa acuminata $\mathrm{L}$. & Biyu Kunti & Leaf, fruit & Herb & 84 & High \\
\hline Musa acuminata L. & Biyu Kayu & Leaf, fruit & Herb & 48 & Moderate \\
\hline Musa acuminata L. & Biyu Bunga & Leaf fruit & Herb & 54 & High \\
\hline Musa acuminata L. & Biyu Keladi & Leaf, fruit & Herb & 99 & High \\
\hline Musa acuminata $\mathrm{L}$. & Biyu Dak Sangket & Leaf, fruit & Herb & 84 & High \\
\hline Musa acuminata colla & Biyu Alas & Fruit & Herb & 32 & Moderate \\
\hline Myrtaceae & & & & & \\
\hline Psidium guajava $\mathrm{L}$. & Nyambu Kristal & Fruit & Shrub & 4 & Very low \\
\hline Syzygium polyanthum Walp. & Don Juwet & Leaf & Tree & 4 & Very low \\
\hline Nyctaginaceae & & & & & \\
\hline Bougenvillea spectabilis L. & Bunga Kertas & Flower & Shrub & 4 & Very low \\
\hline Pisonia alba Span. & Dagdag See & Leaf & Shrub & 6 & Low \\
\hline Oleaceae & & & & & \\
\hline Nyctanthes arbotristis L. & Srigading & Flower & Shrub & 102 & Very high \\
\hline
\end{tabular}




\begin{tabular}{|c|c|c|c|c|c|}
\hline \multicolumn{6}{|l|}{ Oxalidaceae } \\
\hline Averrhoa carambola $\mathrm{L}$. & Belimbing Sayur & Leaf, fruit & Tree & 6 & Low \\
\hline \multicolumn{6}{|l|}{ Pandanaceae } \\
\hline Pandanus amiryllicolius Roxb. & Pandan Arum & Leaf & Shrub & 8 & Low \\
\hline Pandanus tectorius Parkinson ex Du Roi & Pandan Duri & Leaf & Shrub & 12 & Low \\
\hline \multicolumn{6}{|l|}{ Pinaceae } \\
\hline Pinus merkusii Jungh.\& de Vriese & Cemara & Leaf & Tree & 6 & Low \\
\hline \multicolumn{6}{|l|}{ Piperaceae } \\
\hline Piper betle L. var.nigra & Base Bali & Leaf & Herb & 24 & Moderate \\
\hline Piper betle L. & Base biasa & Leaf & Herb & 114 & Very high \\
\hline Piper retrofractum Vahl. & Tabia Bun & Fruit & Herb & 24 & Moderate \\
\hline \multicolumn{6}{|l|}{ Poaceae } \\
\hline Brachiaria mutica (Forssk.) Stapf. & Padang Guwun & Stem+leaf & Herb & 18 & Low \\
\hline Coix lacryma jobi $\mathrm{L}$. & Jali-Jali & Fruit & Herb & 6 & Low \\
\hline Cymbopogon citratus DC & See & Stem & Herb & 4 & Very low \\
\hline Gigantochloa apus (Schult.) Kurz & Tiying Tali & Stem & Tree & 8 & Low \\
\hline Hordeum scalinum Schreb. & Ikuh bojog & Flower & Herb & 12 & Low \\
\hline Imperata cylindrica $\mathrm{L}$. & Ambengan & Leaf & Herb & 18 & Low \\
\hline Oryza sativa $\mathrm{L}$ & Beras & Seed & Herb & 108 & Very high \\
\hline Oryza nivara $\mathrm{L}$ & Beras merah & Fruit, seed & Herb & 39 & Moderate \\
\hline Oryza sativa $\mathrm{L}$. var. glutinosa & Ketan Putih & Fruit, seed & Herb & 70 & High \\
\hline Oryza sativa $\mathrm{L}$. var. glutinosa & Ketan barak & Fruit, seed & Herb & 75 & High \\
\hline Oryza sativa $\mathrm{L}$. var. glutinosa & Injin & Fruit, seed & Herb & 75 & High \\
\hline Oryza sativa $\mathrm{L}$. & Padi Gaga & Fruit & Herb & 12 & Low \\
\hline Oryza sativa $\mathrm{L}$. & Padi Bali & Fruit, seed & Herb & 18 & Low \\
\hline Saccharum officinarum $\mathrm{L}$ & Tebu Guwak & Stem & Herb & 6 & Low \\
\hline Sorghum bicolor $\mathrm{L}$. & Jagung Beleleng & Seed & Herb & 6 & Low \\
\hline Zea mays $\mathrm{L}$. & Jagung & Seed & Herb & 6 & Low \\
\hline \multicolumn{6}{|l|}{ Pteridaceae } \\
\hline Adiantum pedatum L. & Paku condong & Leaf & Herb & 6 & Low \\
\hline \multicolumn{6}{|l|}{ Rubiaceae } \\
\hline Gardenia jasminoides J.Ellis & Jempiring & Flower & Shrub & 4 & Very low \\
\hline Ixora coccinea $\mathrm{L}$. & Jaum-Jaum & Flower & Shrub & 6 & Low \\
\hline Psychotria micrantha Kunth. & Wisnu & Leaf & Shrub & 6 & Low \\
\hline \multicolumn{6}{|l|}{ Rosaceae } \\
\hline Malus domestica Borkh. & Apel & Fruit & Tree & 20 & Moderate \\
\hline Pyrus communis $\mathrm{L}$. & Pir & Fruit & Tree & 22 & Moderate \\
\hline \multicolumn{6}{|l|}{ Rutaceae } \\
\hline Citrus amblycarpa Hassk & Limo & Fruit, leaf & Shrub & 4 & Very low \\
\hline Citrus grandis $\mathrm{L}$. & Jerungga & Fruit & Tree & 12 & Low \\
\hline Citrus reticulata Blanco & Sumaga & Fruit & Shrub & 24 & Moderate \\
\hline Citrus sinensis L. & Juuk & Fruit & Tree & 24 & Moderate \\
\hline Murraya paniculate $\mathrm{L}$. & Kemoning & Leaf & Shrub & 24 & Moderate \\
\hline \multicolumn{6}{|l|}{ Santalaceae } \\
\hline Santalum album $\mathrm{L}$. & Cenana & Stem & Tree & 6 & Low \\
\hline \multicolumn{6}{|l|}{ Sapindaceae } \\
\hline Cardiospermum halicacabum Linn. & Kesuman Jai & Leaf & Herb & 6 & Low \\
\hline Nephelium lappaceum L. & Buluan & Fruit & Tree & 18 & Low \\
\hline \multicolumn{6}{|l|}{ Sapotaceae } \\
\hline Manilkara zapota $\mathrm{L}$. & Sabo & Fruit & Tree & 4 & Very low \\
\hline \multicolumn{6}{|l|}{ Solanaceae } \\
\hline Solanum melongena $\mathrm{L}$. & Tuwung & Fruit & Shrub & 6 & Low \\
\hline \multicolumn{6}{|l|}{ Urticaceae } \\
\hline Laportea stimulans & Lateng Kebo & Leaf & Herb & 6 & Low \\
\hline \multicolumn{6}{|l|}{ Vitaceae } \\
\hline Vitis vinifera $\mathrm{L}$. & Anggur & Fruit & Shrub & 4 & Very low \\
\hline \multicolumn{6}{|l|}{ Zingiberaceae } \\
\hline Alpinia galanga $\mathrm{L}$. & Langkuas & Rhizome & Herb & 57 & High \\
\hline Curcuma longa Linn. & Kunyit & Rhizome & Herb & 18 & Low \\
\hline Zingiber officinale Rosc. & Jahe & Rhizome & Herb & 30 & Moderate \\
\hline
\end{tabular}




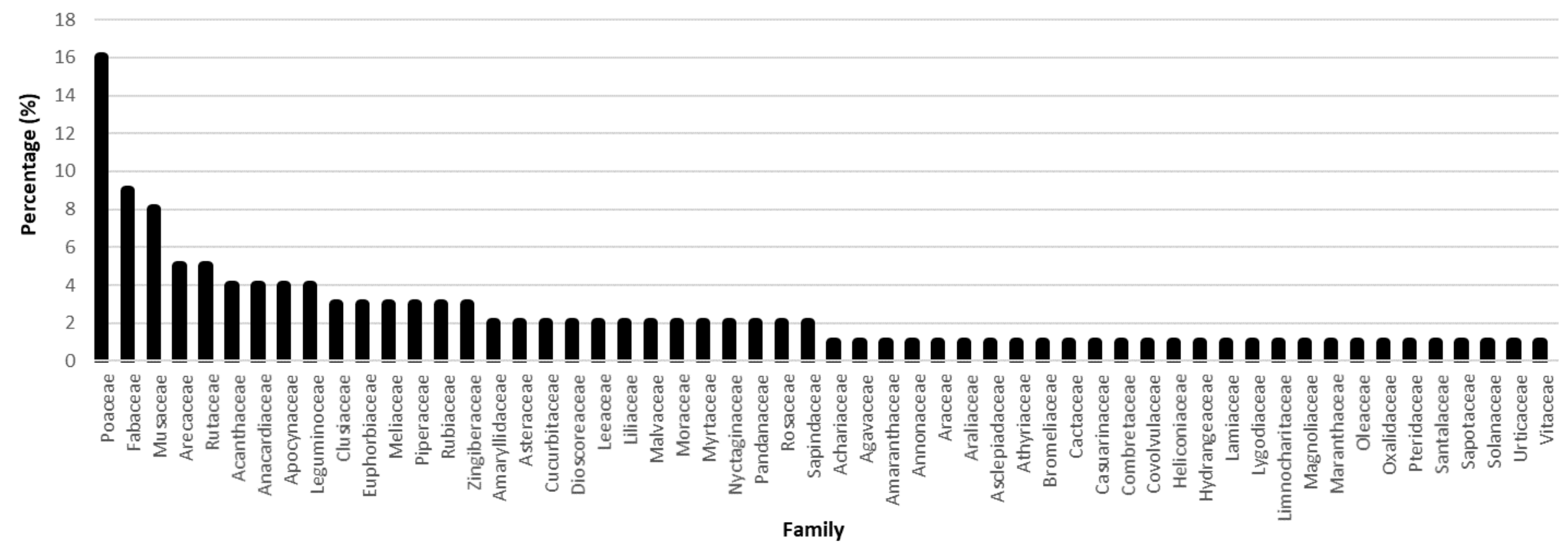

Figure 2. Ngusaba ceremony plant family used by Tenganan Pegringsingan community, Karangasem District, Bali, Indonesia 


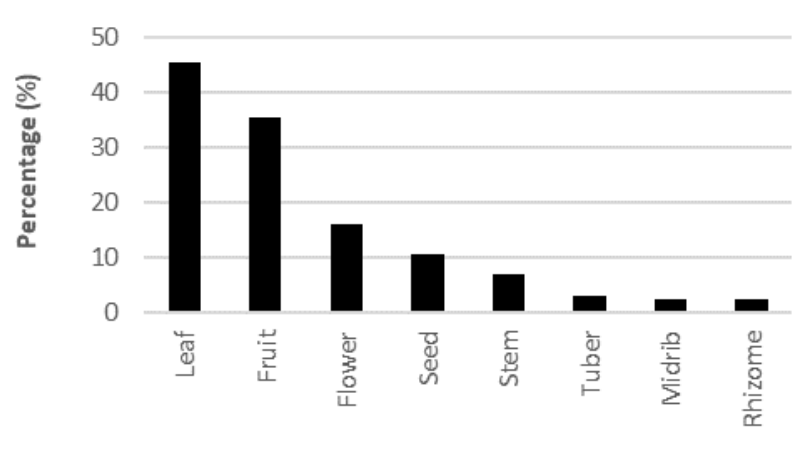

- Plant Parts

Figure 3. Parts of plant used for Ngusaba ceremony by Tenganan Pegringsingan community, Karangasem District, Bali, Indonesia

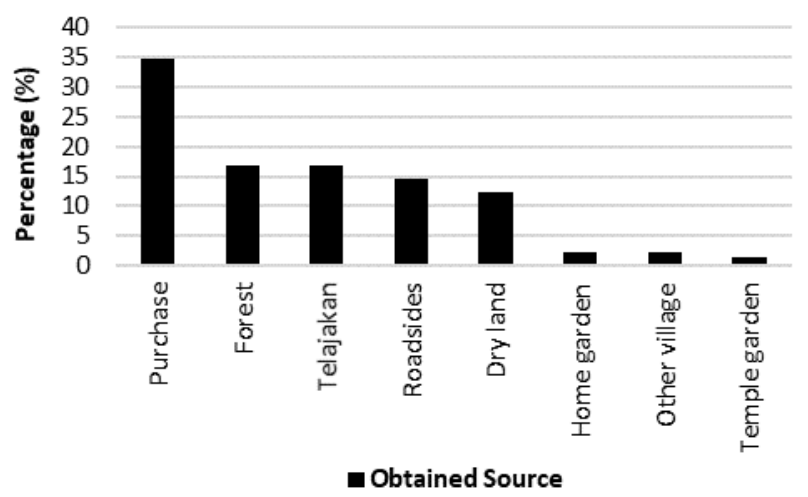

Figure 4. Sources of Ngusaba ceremonial plants by Tenganan Pegringsingan community, Karangasem District, Bali, Indonesia

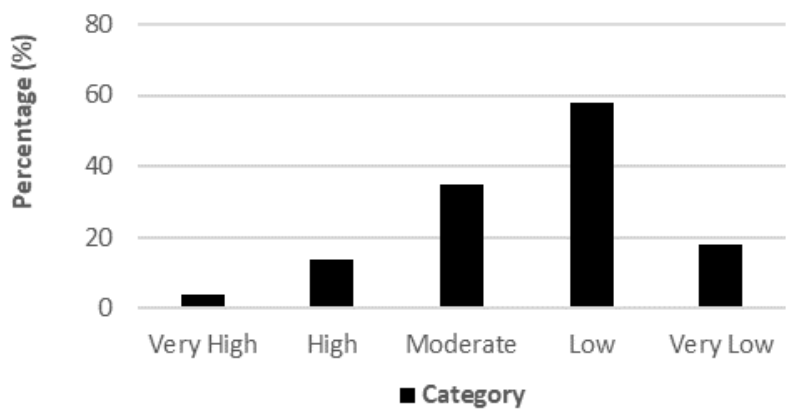

Figure 5. The percentage of the Ngusaba ceremony plants used by Tenganan Pegringsingan community, Karangasem District, Bali, Indonesia

This result showed that the Tenganan Pegringsingan community has the most interaction with the Piper betle L., meaning that this plant species will be used continuously in as much it is in line with the local community's cultural development. The variety of beneficial plants to a community group highly determines the conservation efforts made. The ICS results of Useful Plants as a quantitative ethnobotany analysis showed each useful plant species importance based on community needs (Munawaroh et al. 2011), hence determining the ones to be preserved (Supiandi 2019). The ICS plants' high index indicates a conservation stimulus, such as nature, benefits, and community willingness towards making efforts to develop it. The Tenganan Pegringsingan community tends to provide species that are often conserved as they are typical and cannot be replaced by other plants. In the beginning, local village communities made use of their natural resources and environment primarily based on local knowledge and/or beliefs embedded in their culture (Iskandar 2016). Therefore, human culture can be understood as the knowledge that contains several sets of models used effectively to interpret, understand, and guide behavior in adapting to the environment (Ahimsa-Putra 2012). This situation requires thoughts and efforts on plant 
reintroduction, which the community continuously utilizes by creating a Ngusaba ceremonial plants' garden.

In conclusion, we indicated that Tenganan Pegringsingan people utilize a large number of plant species (130) named and explained for Ngusaba ceremonies. The plant's largest family (16) is Poaceae, while the most widely used part is the leaf, and the highest proportion was obtained by purchasing. Even though most of them are harvested from the wild vegetation, areas such as roadsides, forests, and dry lands are exposed to many threats. The Index of Cultural Significance of the Ngusaba in Tenganan Pegringsingan Village ranges from 2 to 114 , and Piper betle L. has the highest value (114). There is an imbalance between their existence and the use of plants by the community. Many of them become increasingly hard to be found and some have not been found in Tenganan Pegringsingan, such as Musa acuminata L. (biyu kunti), Musa acuminata L.(biyu kayu), Oryza nivara, Oryza sativa var. glutinosa (red and black), Citrus grandis L., Limnocharis flava L., Pinus merkusii L., and Hordeum scalinum Schreb. Therefore, urgent efforts on plant reintroduction are needed to be continuously utilized by the community by creating a Ngusaba ceremonial plants' garden.

\section{ACKNOWLEDGEMENTS}

The authors are grateful to all people in the studied village for their kind hospitality, the share of knowledge on plants and provision of the opportunity and facilities needed in Tenganan Pegringsingan, Manggis Subdistrict, Karangasem Bali, Indonesia.

\section{REFERENCES}

Adiputra N. 2011. Medicinal plants, ceremonial plants and environmental conservation. Bumi Lestari J Environ 11 (2): 346-354. [Indonesian]

Adom D. 2018. Traditional cosmology and nature conservation at the Bomfobiri Wildlife Sanctuary of Ghana. Nat Conserv Res 3 (1): 35 57. DOI: $10.24189 /$ ncr.2018.005

Ahimsa-Putra HS. 2012. Phenomenology approach to understanding religion. Walisongo 20 (2): 271-304. DOI: 10.21580/ws.20.2.200 [Indonesian]

Anderson MJ, Christ TO, Chase JM, Vellend M, Brian D, Inouye, Freestone AL, Sanders NJ, Cornell HV, Comita LS, Davies KF, Harrison SP, Kraft NJB, Stegen JC, Swenson NG. 2011. Navigating the multiple meanings of diversity: A roadmap for ecologists. Ecol Lett 14 (1): 19-28. DOI: 10.1111/j.1461-0248.2010.01552.x.

Arwati M. 2007. Ngusaba Ceremony. Office of Religion of Bali Province, Denpasar. [Indonesian]

Aswani S, Lemahieu A, Sauer WHH. 2018. Global trends of local ecological knowledge and future implications. PLoS ONE 13 (4): e0195440. DOI: 10.1371/journal.pone.0195440

Bosworth K, Lysbeth F, Diley HMA. 2011. School climate factors contributing to student and faculty perceptions of safety in select Arizona schools. J School Health 81 (4): 194-201. DOI: 10.1111/j.1746-1561.2010.00579.x

Caneva G, Traversetti L, Sujarwo W, Zuccarello V. 2017. Sharing ethnobotanical knowledge in traditional villages: Evidence of food and nutraceutical B core groups in Bali, Indonesia. J Econ Bot 71 (4) 303-313. DOI: 10.1007/s12231-017-9395-x.

Constant NL, Tshisikhawe MP. 2018. Hierarchies of knowledge: Ethnobotanical knowledge, practices and beliefs of the Vhavenda in
South Africa for biodiversity conservation. J Ethnobiol Ethnomed 14 (56): 2-28. DOI: 10.1186/s13002-018-0255-2.

Cuadra VP, Cambi V, Ruda MA. 2012. Consequences of the loss of traditional knowledge: The risk of injurious and toxic plants growing in kindergartens. Ethnobot Res App 10: 77-94.

Erawan TS, Alillah AN, Iskandar J. 2018. Ethnobotany of traditional rituals in the Karangwangi Village Cianjur District, West Java, Indonesia. Asian J Ethnobiol 1 (2): 52-60. DOI: 10.13057/asianjethnobiol/y010201.

Geng Y, Hu G, Ranjitkar S, Shi X, Zhang Y, Wang Y. 2017. The implications of ritual practices and ritual plant use on nature conservation: a case study among the Naxi in Yunnan Province, Southwest China. J Ethnobiol Ethnomed 13 (1): 1-11. DOI: 10.1186/s13002-017-0186-3.

Gomez BE, Mingoria S Reyes GV, Calvet LMontes C. 2010. Traditional ecological knowledge trends in the transition to a market economy: Empirical study in the Don Ana Natural Areas. Conserv Biol 24 (3): 721-729. DOI: 10.1111/j.1523-1739.2009.01401.x.

Hager TF. 2008. Processing and storage effects on monomeric anthocyanins, percent polymeric color, and antioxidant capacity of processed blackberry products. J Agric Food Chem 56 (3): 689-695. DOI: $10.1021 / \mathrm{j}$ f071994g.

Hakim L. 2014. Ethnobotany and home garden management: Food security, health, and agro-tourism. Selaras, Malang. [Indonesian]

Handayani A. 2015. Utilization of medical plants by people around Mount Simpang Nature Reserve, West Java. Pros Sem Nas Masy Biodiv Indon 1 (6): 1425-1432. [Indonesian]

Hariyadi B, Ticktin T. 2012. Uras: Medicinal and ritual plants of Serampas, Jambi Indonesia. J Ethnobot Res Appl 10: 133149.

Helida A, Zuhud EAM, Hardjanto, Purwanto, Hikmat A. 2015. Index of cultural significance as a potential tool for conservation of plants diversity by communities in The Kerinci Seblat National Park. Jurnal Manajemen Hutan Tropika 21 (3): 192-201. DOI: 10.7226/jtfm.21.3.192. [Indonesian]

Iskandar J. 2016. Ethnobiology and cultural biodiversity in Indonesia. Indones J Anthropol 1 (1): 27-42.

Iskandar J, Iskandar BS. 2017. Various plants of traditional rituals: Ethnobotanical research among Baduy community. Biosaintifica 9 (1): 114-125. DOI: 10.15294/biosaintifika.v9i1.8117. [Indonesian]

Ju Y, Zhuo J, Lui B, Long C. 2013. Eating from the wild: Diversity of wild edible plants used by Tibetans in Shangrila-Region, Yunnan, China. J Ethnobiol Ethnomed 9 (1): 1-22. DOI: 10.1186/1746-42699-28

Kakudidi EK. 2004. Cultural and social uses of plants from and around Kibale National Park, Western Uganda. Afr J Ecol 42 (1): 114-118. DOI: 10.1111/j.1365-2028.2004.00472.x.

Mesfin K, Tekle G, Tesfay T. 2013. Ethnobotanical study of traditional medicinal plants used by indigenous people of Gemad District, Northern Ethiopia. J Med Plants Stud 1 (4): 32-37.

Monograph of Tenganan Village, Karangasem. 2020.

Munawaroh E, Saparita R, Purwanto Y. 2011. Community dependence on non-timber forest products in Malinau, East Kalimantan: an ethnobotany analysis and its implications for forest conservation. Berkala Hayati Special Edition 7A: 51-58.

Navia ZI, Audira D, Afifah N, Turnip K, Nuraini, Suwardi AB. 2020. Ethnobotanical investigation of spice and condiment plants used by the Taming tribe in Aceh, Indonesia. Biodiversitas 21 (10): 44674473. DOI: $10.13057 /$ biodiv/d211001.

Nisyapuri FF, Johan I, Ruhyat P. 2018. Ethnobotany of medicinal plants in Wonoharjo Village, Pangandaran Regency, West Java. Biodiversity 4 (2): 122-132. DOI: $10.13057 / \mathrm{psnmbi} / \mathrm{m} 040205$.

Nurdiani N. 2014. Snowball sampling techniques in dry land research. Comtech 5 (2): 1110-1118.

O'Neill AR, Badola HK, Dhyani PP, Rana SK. 2017. Integrating ethnobiological knowledge into biodiversity conservation in the eastern Himalayas. J Ethnobiol Ethnomed 13 (1): 1-14. DOI: 10.1186/s13002-017-0148-9.

Pieroni A, Anely N, Avni H, Mustafa B, Bruno S, Kevin C, Cassandra LQ. 2014. Local knowledge on plant and domestic remedies in the mountain village of Peshkopia (Eastern Albania). J Mt Sci 11 (1): 180-194. DOI: 10.1007/s11629-013-2651-3.

Purwanto Y. 2003. Ethnobotany Research Methods. Lab. Ethnobotany. Balitbang Botani, LIPI Biology Research and Development Center, Bogor, Indonesia. [Indonesian]

Putri NS, Dewi R, Fitriana. 2017. Ceremony bridal process in Teubang Phui Baru village, Montasik sub-district, Aceh Besar district. Jurnal 
Ilmiah Mahasiswa Pendidikan Kesejahteraan Keluarga 2 (4): 42-57. [Indonesian]

Ristanto RH, Suryanda A, Rismayati AI, Rimadana A, Datau R. 2020 Ethnobotany: a plant of Hindu-Balinese religious rituals. Jurnal Pendidikan Biologi 5 (1): 96-105. DOI: 10.31932/jpbio.v5i1.642 [Indonesian]

Setiawan H, Qiptiyah M. 2014. Ethnobotany study of the Moronene tribe in the Rawa Aopa Watumohai National Park. Wallacea J Res 3 (2): 107-117. DOI: 10.18330/jwallacea.2014.vol3iss2pp107-117.

Sharma UK, Pegu S. 2011. Ethnobotany of religious and supernatural beliefs of the Mising tribes of Assam with special reference to the 'Dobur Uie'. J Ethnobiol Ethnomed 7 (1): 1-13. DOI: 10.1186/17464269-7-16.

Sheybani H, Charmchiyan M, Azadboni ZB. 2015. Factors affecting villagers' participation in forest conservation in the region of Miyandoroud. Indian J Fundam Appl Life Sci 5 (S1): 3724-3730.

Sujarwo W, Caneva G. 2016. Using quantitative indices to evaluate the cultural importance of food and nutraceutical plants: Comparative data from the Island of Bali (Indonesia). J Cul Her 18: 342-348. DOI: 10.1016/j.culher.2015.06.006.

Sujarwo W, Keim AP, Caneva G, Tonia C, Nicoletti M. 2016 Etnobotanical uses of neem (Acadiractha Indica A. Juss.; Meliaceae) leaves in Bali (Indonesia) and the Indian subcontinent in relation with historical background and phytochemical properties. J Ethnopharmacol 189: 186-193. DOI: 10.1016/j.jep.2016.05.014.

Sujarwo W, Keim AP. 2017. Ethnobotanical study of traditional building materials from the Island of Bali, Indonesia. Econ Bot 71 (3): 224240. DOI: $10.1007 / \mathrm{s} 12231-017-9385-\mathrm{z}$.

Sujarwo W, Caneva G, Zuccarello V. 2020. Patterns of plant use in religious offerings in Bali (Indonesia). Acta Bot Bras 34 (1): 40-53. DOI: $10.1590 / 0102-33062019 a b b 0110$

Supiandi MI, Mahanal S, Zubaidah S, Julung HBEGE. 2019. Ethnobotany of traditional medicinal plants used by Dayak Desa community in Sintang, West Kalimantan, Indonesia. Biodiversitas 20 (5): 12641270. DOI: $10.13057 /$ biodiv/d200516.
Surata IK, Gata IW, Sudiana IM. 2015. Ethnobotanical study of Balinese Hindu ceremonial plants as an effort to protect local wisdom. Jurnal Kajian Bali 5 (2): 265-284. [Indonesian]

Suryadarma IGP. 2017. Building the character of independence of an ethnobiological approach to local uniqueness in national perspectives and global reflections. Biotic National Seminar Proceedings. Yogyakarta, November 26, 2017. [Indonesian]

Sutrisno IH, Akob B, Navia ZI, Nuraini, Suwardi AB, 2020. Documentation of ritual plants used among the Aceh Tribe in Peureulak, East Aceh District, Indonesia. Biodiversitas 21 (11): 49904999. DOI: $10.13057 /$ biodiv/d211102.

Suwardi AB, Navia ZI, Harmawan T, Syamsuardi, Mukhtar E. 2020. Ethnobotany and conservation of indigenous edible fruit plants in South Aceh, Indonesia. Biodiversitas 21 (5): 1850-1860. DOI: 10.13057/biodiv/d210511.

Tamalene MN, Al Mudhar MHI, Suarsini E, Rahman F, Hasan S. 2016 Ethnobotany of Canarium plant species used by Tobelo Dalam (Togutil) ethnic community of Halmahera Island, Indonesia. Biodiversitas 17 (1): 61-69. DOI: 10.13057/biodiv/d170109.

Tapundu AS, Anam S. 2015. Studi etnobotani tumbuhan obat pada Suku Seko di Desa Tanah Harapan Kabupaten Sigi, Sulawesi Tengah. Jurnal Biocelebes 9 (2): 66-86. [Indonesian]

Turner NJ. 1998. The importance of a rose: Evaluating the cultural significance of plants in Thompson and Lillooet Interior Salish. J Amer Anthropol 90 (2): 272-290. DOI: 10.1525/aa.1988.90.2.02a00020.

Yusro, Fathul, Yeni M, Farah D, Kazuhiro. 2014. Inventory of medicinal plants for fever used by four Dayak Sub Ethnic in West Kalimantan, Indonesia. Kuroshio Sci 8 (1): 33-38.

Vásquez A, Vibrans H, Vergara SF, Caballero J. 2016. Intracultural differences in local botanical knowledge and knowledge loss among the Mexican Isthmus Zapotecs. PLoS ONE 11 (3): e0151693. DOI: 10.1371/journal.pone.0151693. 